\title{
Predictive Model for Secundum Atrial Septal Defect Closure with Pulmonary Artery Hypertension in Adult: When to Close
}

\author{
Supomo, MD, PhD,${ }^{1}$ Adika Zhulhi Arjana, $\mathrm{MD},{ }^{2}$ Handy Darmawan, $\mathrm{MD}^{3}$ \\ ${ }^{1}$ Cardiothoracic and Vascular Surgeon, Dr. Sardjito Hospital, ${ }^{2}$ Faculty of Medicine, Universitas Islam Indonesia, ${ }^{1,3}$ Faculty of \\ Medicine, Universitas Gadjah Mada, Yogyakarta, Indonesia
}

\section{ABSTRACT}

Background: Secundum atrial septal defect (SASD) closure is contraindicated in the presence of severe pulmonary artery hypertension $(\mathrm{PAH})$. However, there is no consensus on the threshold of severe $\mathrm{PAH}$, in terms of mean pulmonary artery pressure (mPAP), which would contraindicate for defect closure surgery in adults. Furthermore, PAH can persist, or even increase in severity, after the closure. The aim of this study was to produce a predictive model correlating mPAP in adult SASD patients with $\mathrm{PAH}$ after defect closure surgery.

Methods: Between January 2014 and March 2017, 29 consecutive adult SASD patients who had PAH and underwent defect closure surgery were included in the study. Age, right atrium (RA) dimension, right ventricle (RV) dimension, and $\mathrm{mPAP}$ before surgery were analyzed using multiple regression to produce the model.

Results: Multiple regression produced the following model: $\mathrm{mPAP}$ prediction $=(0.24)($ Age $)+(0.06)(\mathrm{mPAP}$ before surgery $)+(0.17)(\mathrm{RA}$ dimension $)+(0.47)(\mathrm{RV}$ dimension $)-$ $13.79(P=.0008)$. The mPAP prediction was compared to mPAP of the patients six to nine months after surgery, and showed no significant difference $(P=.9562)$.

Conclusion: In adult SASD patients with $\mathrm{PAH}$, our model can significantly predict the mPAP after the closure. If the predicted $\mathrm{mPAP}$ is within its normal range, the closure is indicated.

\section{INTRODUCTION}

Atrial septal defect (ASD) is the second most common congenital heart disease, occurring in 1.64 per 1,000 births, predominantly in females [Van Der Linde 2011]. Secundum atrial septal defect (SASD) composes $75 \%$ of all ASD cases. About $97 \%$ of non-diagnosed ASD patients will survive into adulthood [Kuijpers 2015]. Among all of these adult patients, 35\% develop pulmonary artery hypertension (PAH) [Engelfriet 2008].

Defect closure is contraindicated in the presence of severe PAH [Jung 2013; Schwerzmann 2006]. However, there is no consensus on what threshold value of mean pulmonary artery

Received September 30, 2017; accepted Fanuary 27, 2018.

Correspondence: Supomo, MD, PhD, Cardiothoracic and Vascular Surgery Dr. Sardjito Hospital, Kesehatan street, Sleman, Daerah Istimewa Yogyakarta, Indonesia; +6285727663500 (e-mail: supomo.tkv@mail.ugm.ac.id). pressure (mPAP) is considered severe, and is thus contraindicated for SASD closure surgery in adults [Kozlik-Feldman 2016; Jung 2013; Oliveira 2008; Schwerzmann 2006]. In addition, PAH can endure or even increase in severity after the closure [Engelfriet 2008]. These facts create controversy over when to close the defect, and whether to close the defect in the presence of PAH.

Several factors were proven to affect PAH even after SASD closure. Age, the dimension of the right ventricle (RV), right atrium (RA), and mPAP before surgery all correlated with PAH after SASD closure [Gabriels 2014]. The aim of this study was to produce a predictive model correlating mPAP in adult SASD patients with PAH after defect closure surgery based on these factors.

\section{MATERIALS AND METHOD}

Between January 2014 and March 2017, 67 SASD patients with PAH over 18 years of age underwent defect closure surgery without adjuvant therapies in Dr. Sardjito General Hospital, Yogyakarta, Indonesia. The data of the patients were obtained from medical records, retrospectively. Twenty nine SASD patients ( 4 male and 25 female) with complete required data on their medical record were included in this study. All of the patients underwent SASD closure through median

Table 1. Baseline Characteristics of Patients

\begin{tabular}{lccc}
\hline Variables & $\mathrm{N}(\%)$ & Mean \pm SD & Median (min-max) \\
\hline Male & $4(86.21)$ & & \\
Female & $25(13.79)$ & & \\
Age (years)* & & \\
ASD Diameter $(\mathrm{cm}) *$ & & $0.9-4.2$ \\
mPAP (mmHg) & $30.19 \pm 12.64$ & $31(24-48)$ \\
LA Dimension (mm)* & & \\
RA Dimension (mm) & $45.10 \pm 6.02$ & \\
RV Dimension (mm) & $42 \pm 5.89$ & \\
Ejection Fraction (\%) & $70.31 \pm 6.48$ & \\
\hline
\end{tabular}

SD indicates standard deviation; ASD, atrial septal defect; mPAP, mean pulmonary artery pressure; LA, left atrium; RA, right atrium; RV, right ventricle.

*Non-parametric data. 
Table 2. Characteristic Data Comparison Between Males and Females

\begin{tabular}{lccc}
\hline Variables & Male & Female & $P$ \\
\hline Age (years) & $30 \pm 8.45$ & $34.44 \pm 11.71$ & .5267 \\
Minimum Diameter of Defect $(\mathrm{mm})$ & $2.08 \pm 0.53$ & $2.45 \pm 0.95$ & .4107 \\
Maximum Diameter of Defect $(\mathrm{mm})$ & $2.45 \pm 0.48$ & $2.8 \pm 0.9$ & .3266 \\
LA Dimension (mm) * & $30(30-33)$ & $31(24-48)$ & .6342 \\
RA Dimension $(\mathrm{mm})$ & $45 \pm 4$ & $45.12 \pm 6.35$ & .9713 \\
RV Dimension $(\mathrm{mm})$ & $42.25 \pm 4.35$ & $41.96 \pm 6.18$ & .9292 \\
Ejection Fraction $(\%)$ & $66.5 \pm 8.66$ & $70.92 \pm 6.06$ & .2108 \\
mPAP (mmHg) & $23.5 \pm 3.32$ & $30.97 \pm 13.25$ & .4499 \\
\hline
\end{tabular}

LA indicates left atrium; RA, right atrium; RV, right ventricle; mPAP, mean pulmonary artery pressure.

*Non-parametric data.

Table 3. Bivariate Analysis of Variables Before and After Defect Closure Surgery

\begin{tabular}{lccc}
\hline Variables & Before & After & $P$ \\
\hline LA Dimension (mm)* & $31(24-48)$ & $33(25-47)$ & .1824 \\
RA Dimension (mm) & $45.10 \pm 6.02$ & $37.28 \pm 5.32$ & $<.0001$ \\
RV Dimension (mm) & $42.00 \pm 5.89$ & $31.83 \pm 4.34$ & $<.0001$ \\
Ejection Fraction (\%) & $70.31 \pm 6.48$ & $70.21 \pm 5.37$ & .9304 \\
mPAP (mmHg) & $30.40 \pm 12.45$ & $24.78 \pm 6.99$ & .0315 \\
\hline
\end{tabular}

LA indicates left atrium; RA, right atrium; RV, right ventricle; mPAP, mean pulmonary artery pressure

*Non-parametric data.

sternotomy and direct suture of the defect. Follow-up of the patients was performed six to nine months after surgery. Evaluation of the patients was performed using transthoracic echocardiography. The institutional ethics committee of the Medical Faculty of the Universitas Gadjah Mada approved this study, and the need for individual consent was waived.

All of the data were analyzed using Medcalc software. Characteristics of the patients before the surgery are presented in Table 1. Continuous and categorical variables are presented along with mean \pm standard deviation (SD) and percentage, respectively. Continuous variables with non-parametric data were presented using the median. Characteristic data of males and females were compared using an unpaired student's t-test in order to prove that there was no significant difference between these variables, even though the majority of the subjects were female (Table 2). The Mann Whitney test was performed for variables with non-parametric data. The results are considered to be significant if the $P<.05$. Bivariate analysis using a paired student's t-test was performed to test the difference of all variables before, and six to nine months

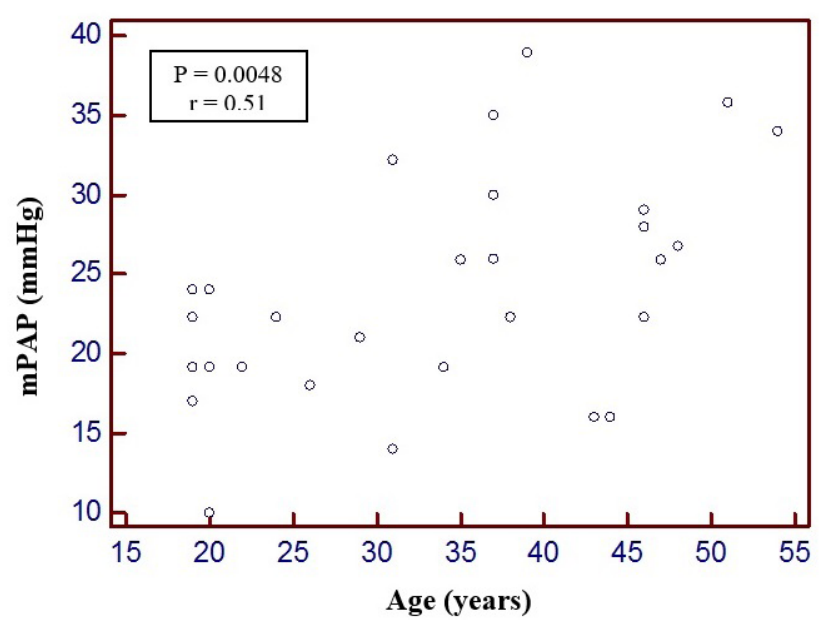

Figure 1. Scatterplot of spearman's rank correlation test between age and mPAP six to nine months after defect closure surgery.

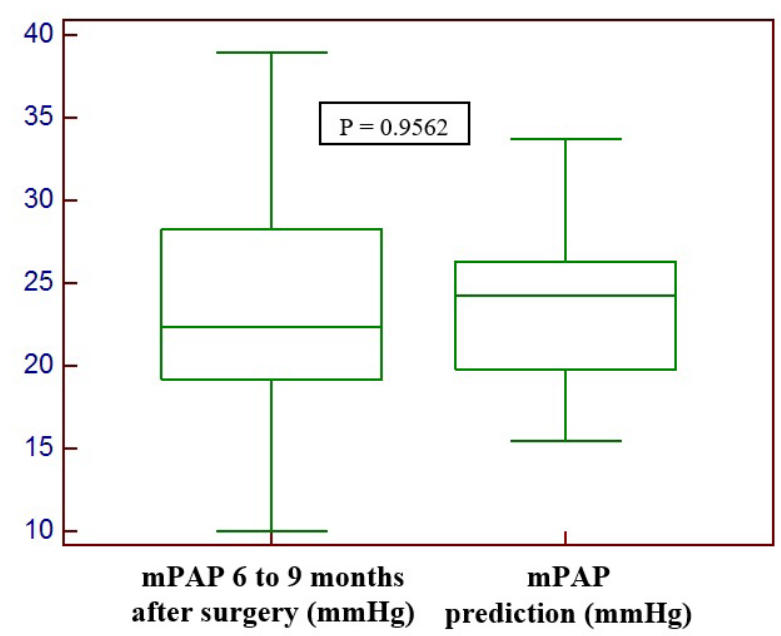

Figure 2. Comparison between mPAP prediction and mPAP after six to nine months of follow up showing no significant difference $(P=.9562)$.

after, the surgery (Table 3). The Wilcoxon test was performed for variables with non-parametric data. Factors that showed significant difference were included for multiple regression to produce the mPAP prediction models. The MPAP prediction and the mPAP of the patients after surgery, during follow-up, were compared using an unpaired student's t-test to calculate the difference (Figure 2).

\section{RESULTS}

Characteristic data of the patients before the defect closure surgery are shown in Table 1 . The majority of the subjects were female, making up $86.21 \%$ of the total number of subjects. However, comparison of males and females showed no significant difference in age; maximum and minimum diameter of the defect; RV, RA, and left atrium (LA) dimension; ejection fraction; or mPAP (Table 2). 
Bivariate analysis of RV, RA, and LA dimension, ejection fraction, and mPAP before and six to nine months after defect closure surgery are shown in Table 3. The LA dimension and ejection fraction showed no significant difference before or after defect closure surgery $(P=.1824$ and $P=.9304$, respectively). On the other hand, $\mathrm{RV}$ dimension, $\mathrm{RA}$ dimension, and mPAP showed a significant difference $(P<.0001, P<.0001$, and $P=.0315$, respectively). Spearman's rank correlation test was performed using age and mPAP of the patient after defect closure surgery. This showed significant correlation, with $\mathrm{r}=0.51$ and $P=.0048$ (Figure 1). These variables were included in multiple regression to produce a predictive model of mPAP after defect closure surgery in adult SASD patients with PAH.

Multiple regression produced the following model: mPAP prediction $=(0.24)(\mathrm{Age})+(0.06)(\mathrm{mPAP}$ before surgery $)$ $+(0.17)(\mathrm{RA}$ dimension $)+(0.47)(\mathrm{RV}$ dimension $)-13.79$ $(P=.0008)$. There was no significant difference between predicted mPAP and mPAP of the patients after six to nine months during follow up with $P=.9562$ (Figure 2).

\section{DISCUSSION}

Defect closure surgery is contraindicated for SASD patients in the presence of severe PAH [Jung 2013; Schwerzmann 2006]. However, there are differences regarding the suggested threshold of PAH severity for the contraindication of surgery in adults. Oliveira et al stated that the patients with $\mathrm{mPAP}>65 \mathrm{mmHg}$ are diagnosed as having severe $\mathrm{PAH}$ [Oliveira 2008]. On the other hand, Schwerzmann et al and Jung et al considered patients with $\mathrm{mPAP}$ of $53 \mathrm{mmHg}$ and 50 $\mathrm{mmHg}$, respectively, as having severe PAH [Jung 2013; Schwerzmann 2006]. Though Kozlik-Feldman et al presented an algorithm for management of SASD based on pulmonary vascular resistance (PVR), it is only for use in pediatric patients [Kozlik-Feldman 2016].

A previous study by Humenberger et al showed immediate reduction of $\mathrm{mPAP}$ and $\mathrm{RV}$ dimension in all patients in their study after SASD closure [Humenberger 2011]. This reduction after surgery occurred due to the significant decrease of transpulmonary flow. However, a recent large prospective cohort by Engelfriet et al showed that the PAH remained in $13 \%$ of adult SASD patients after defect closure surgery [Engelfriet 2008]. Gabriels et al presented similar results [Gabriels 2014]. The difference of PAH in ASD patients after defect closure compared to patients without defect closure in their study was not significant, with a prevalence of $13.3 \%$ and $15.9 \%$, respectively. These conflicting results raise questions over when to close the defect, and whether or not to close it at all.

Persistence or subsequent development of PAH after defect closure surgery is affected by several factors. Gabriels et al revealed age to be an independent predictor of $\mathrm{PAH}$ after defect closure surgery with a cutoff point of 54 years, which had almost $100 \%$ negative predictive value [Gabriels 2014]. Older patients had a worse clinical outcome after defect closure due to previous chronic overflow and volume overload on pulmonary circulation. This phenomenon produced irreversible changes of the pulmonary artery, and persistence of PAH after defect closure surgery [Gabriels 2014; Sachweh 2006]. The current study showed a corresponding result of significant correlation between age and mPAP after surgery (Figure 1).

Dilatation of RA and RV correlated with the deterioration of clinical outcome in PAH [Grapsa 2012]. These factors also correlated with persistence of PAH after defect closure surgery [Gabriels 2014]. The current study showed a significant difference in RA and RV dimension before and after defect closure surgery, as well as a significant difference in mPAP (Table 3). This finding is consistent with a study by Yong et al, which observed right heart enlargement, persistent symptoms, and residual PAH after defect closure surgery in those patients with higher baseline mPAP [Yong 2009].

The current study produced a predictive model of mPAP after defect closure surgery in adult SASD patients with $\mathrm{PAH}$ using multiple regression of the significant findings. The model produced predicted $\mathrm{mPAP}$, which showed no significant difference from the actual mPAP of the patient six to nine months after defect closure surgery (Figure 2). Therefore, predicted mPAP can be used as a consideration when deciding when to close and whether to close the defect in the case of PAH. If the predicted mPAP is within its normal range $(<25 \mathrm{mmHg})$, the closure is indicated [Jung 2013; Oliveira 2008; Schwerzmann 2006]. Age, RA dimension, RV dimension, and mPAP before defect closure surgery were predictors of $\mathrm{PAH}$ after defect closure surgery. The predictive model of mPAP after surgery produced by multiple regression of these factors can significantly predict the mPAP after defect closure surgery.

\section{REFERENCES}

Engelfriet P, Meijboom F, Boersma E, et al. 2008. Repaired and open atrial septal defect type II in adulthood: an epidemiological study of a large european cohort. Int J Cardiol 126:379-85

Gabriels C, De Meester P, Pasquet A, et al. 2014. A different view on predictors of pulmonary hypertension in secundum atrial septal defect. Int J Cardiol 176:833-40.

Grapsa J, Gibbs SR, Cabrita IZ, et al. 2012. The association of clinical outcome with right atrial and ventricular remodelling in patients with pulmonary arterial hypertension: study with real-time three-dimensional echocardiography. Eur Heart J Cardiovasc Imaging 13:666-72.

Humenberger M, Rosenhek R, Gabriel H, et al. 2011. Benefit of atrial defect closure in adults: impact of age. Eur Heart J 32:553-60.

Jung IH, Lee SY, Lee SJ, et al. 2013. Device closure of a large atrial septal defect in a patient with severe pulmonary arterial hypertension after 1 year use of an oral endothelin receptor antagonist. J Cardiovasc Ultrasound 21:140-4.

Kozlik-Feldman R, Hansmann G, Bonnet D, et al. 2016. Pulmonary hypertension in children with congenital heart disease (PAH-CHD, PPHVD-CHD): expert consensus statement on the diagnosis and treatment of paediatric pulmonary hypertension. Heart 102:ii42-ii48.

Kuijpers JM, Mulder BJM, Bouma BJ. 2015. Secundum atrial septal defect in adults: a practical review and recent developments. Neth Heart J 23:205-11.

Oliveira EC, Amaral CFS, Moura MA, et al. 2008. Testing pulmonar vasoreactivity. J Bras Pneumol 34:838-44. 
Sachweh JS, Daebritz SH, Hermanns B, et al. 2006. Hypertensive pulmonary vascular disease in adults with secundum or sinus venosus atrial septal defect. Ann Thorac Surg 81:207-13.

Schwerzmann M, Zafar M, McLaughlin PR, et al. 2006. Atrial septal defect closure in a patient with "irre $\neg$ versible" pulmonary hypertensive arteriopathy. Int J Cardiol 110:104-7. van der Linde D, Konings EEM, Slager MA, et al. 2011. Birth prevalence of congenital heart disease worldwide: a systematic review and metaanalysis. J Am Coll Cardiol 58:2241-7.

Yong G, Khairy P, De Guise P, et al. 2009. Pulmonary arterial hypertension in patients with transcatheter closure of secundum atrial septal defect: a longitudinal study. Cardiovasc Intervent 2:455-62. 